\section{„In Frankfurt werden wir vieles neu machen!“}

Professor Martin Wiesmann ist Kongresspräsident der 53. Jahrestagung der Deutschen Gesellschaft für Neuroradiologie und wird die erste Jahrestagung im Kap Europa in Frankfurt verantworten. Im Interview skizziert er die Neuerungen für den Kongress-Standort 2018.

\section{Nach neun Jahren Gürzenich zieht der Neurorad 2018 von Köln ins Kap Europa nach Frankfurt - was bedeutet dieser Um- zug für den Kongress?}

Der Kölner Stadtteil Gürzenich war ein wichtiger Ort für den Neurorad - um anzukommen, zu wachsen und zu der „Marke“ zu werden, die der Kongress heute ist. Mit den Jahren aber ist die Location zu klein geworden und hat sich als nicht mehr zeitgemäß für eine moderne Kongressgestaltung erwiesen. Das Kap Europa, das ab

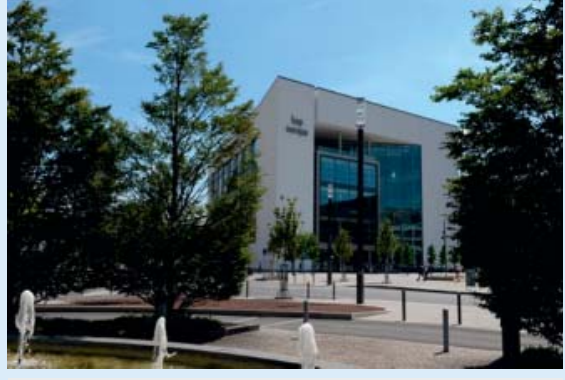

Das Kap Europa Frankfurt, Austragungsort der 53. Jahrestagung der DGNR 2018 (c) Messe Frankfurt

2018 unser Veranstaltungsort sein wird, ist ein hochmodernes Kongresszentrum, das uns die Möglichkeit gibt, den Kongress weiterzuentwickeln - hinsichtlich Teilnehmerzahlen, Workshop-Angeboten, Präsentation der wissenschaftlichen Beiträge, um nur einige Aspekte zu nennen.

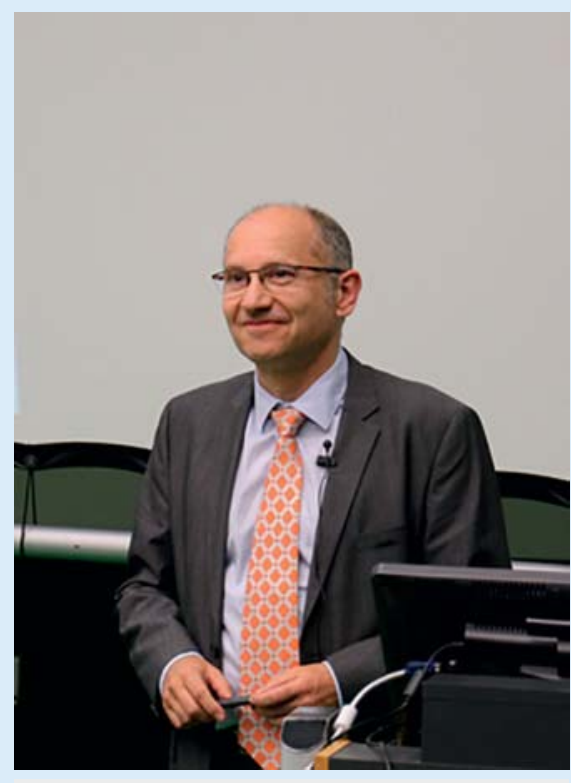

Kongresspräsident Professor Dr. Martin Wiesmann ( UKAachen

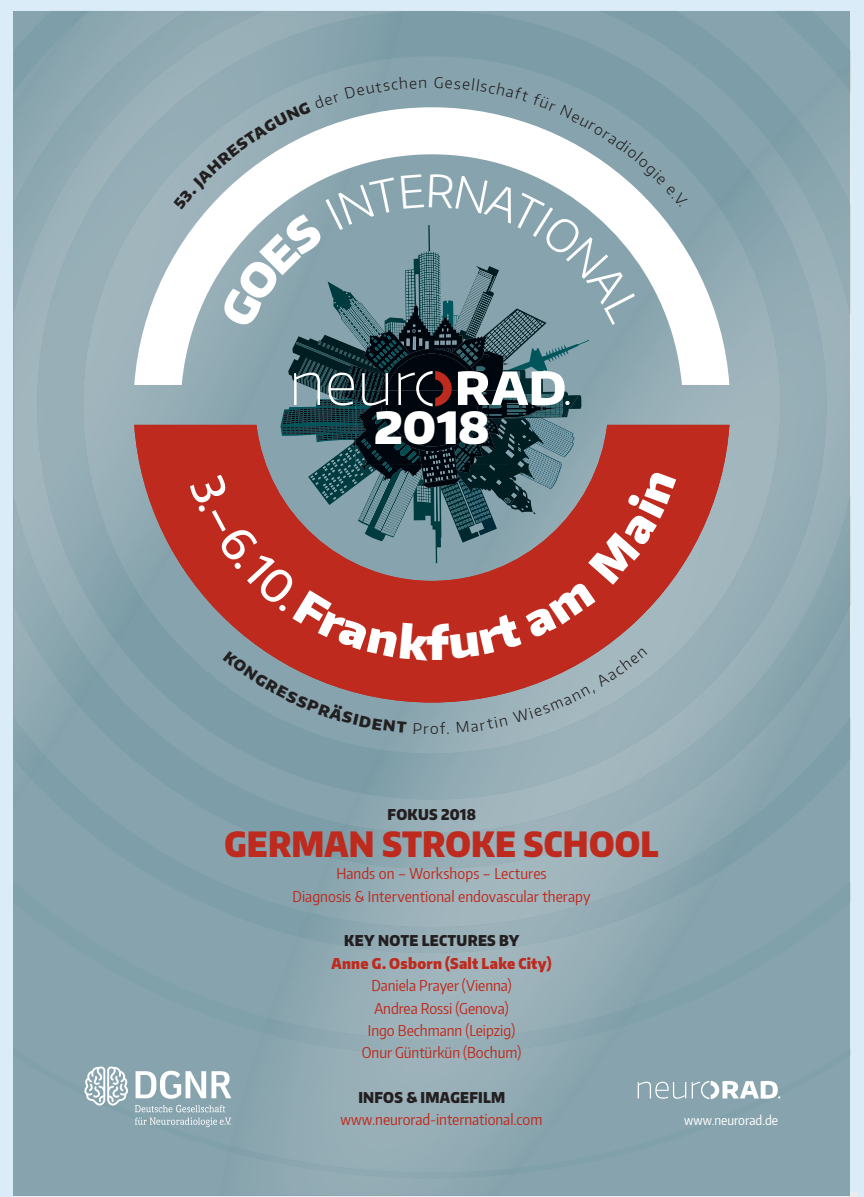

Das Motto des Kongresses lautet: „Neurorad goes international“ - welche Idee steht hinter der Internationalisierung und was wird die Kollegen im Programm hierzu erwarten?

Die deutschen Neuroradiologen sind hochgefragte Referenten im Ausland; man schaut besonders auf die interventionelle Neuroradiologie, die endovaskuläre Schlaganfalltherapie, die maßgeblich in Deutschland entwickelt wurde und zur flächendeckenden Versorgung geführt hat. Da lag die Idee in der Luft, ausländische Kollegen - gerade aus den angrenzenden Ländern zu unserem Kongress nach Deutschland einzuladen. Die „German-Stroke-School“ im Speziellen lädt Kolleginnen und Kollegen zu intensiven, simulatoren-unterstützten Hands-On-Workshops ein. Darüber hinaus bieten wir ein hochattraktives englischsprachiges Vortragsprogramm mit interessanten Referenten, das sicher auch DGNR-Kolleginnen und -Kollegen ansprechen wird. 
Welche Highlights und Innovationen sind für die erste Jahrestagung der DGNR in Frankfurt geplant?

Ein Highlight ist sicherlich der Auftritt von Ann Osborn, die sich sehr freut, nach fünf Jahren erneut nach Deutschland zu kommen. Mit Daniela Prayer aus Wien und Andrea Rossi aus Genua haben wir zwei weitere führende Experten unseres Fachs nach Frankfurt eingeladen. Außerdem werden wir in der Mittagszeit unser Publikum mit knackigen Kurzvorträgen von fachangrenzenden Referenten begeistern. Auch die Präsentation der wissenschaftlichen Einreichungen werden wir neu aufstellen. In Frankfurt werden wir vieles neu machen. Wir werden aber auch Bewährtes, wie die konsequente Ausrichtung des Neurorads als Fortbildungsveranstaltung, fortsetzen und neu beleben.

Neurorad goes international - Welcome to Frankfurt!

Eine englischsprachige Programmschiene, ein hochmodernes Kongresszentrum und eine verkehrstechnisch optimal gelegene Stadt: All das wird die Neurorad-Jahrestagungen ab dem Herbst 2018 auszeichnen. Schwerpunkt des ersten Frankfurter Neurorad wird die German-Stroke-School sein, die in- und ausländischen Teilnehmern eine intensive Hands-on-Fortbildung in der Diagnostik und Therapie beim Schlaganfall vermitteln wird.

Stars der 53. Jahrestagung, die unter der Leitung von Prof. Dr. Martin Wiesmann (Aachen) steht, sind die Keynote-Speaker Anne G. Osborn (Salt Lake City, USA), Andrea Rossi (Genua, Italien) und Daniela Prayer (Wien, Österreich).

Call for Abstract 15.03. - 27.05.2018

www.neurorad-international.com 